\title{
European Extremely Large Telescope: Isopistonic angle measurements at Aklim site
}

\author{
T. Elhalkouj \\ ${ }^{1}$ UFR : APHE, Cadi Ayyad University, \\ Av. Prince My Abdellah, BP 2390 Marrakech, Morocco \\ ${ }^{2}$ CRMEF-Marrakech 40000, Morocco \\ A. Habib \\ ${ }^{1}$ UFR : APHE, Cadi Ayyad University, \\ Av. Prince My Abdellah, BP 2390 Marrakech, Morocco \\ ${ }^{2}$ CRMEF-Marrakech, 40000, Morocco \\ My.Y. Elazhari \\ ${ }^{1}$ UFR : APHE, Cadi Ayyad University, \\ Av. Prince My Abdellah, BP 2390 Marrakech, Morocco \\ 2 CNIPE, MENFCRS, Rabat
}

\author{
M. Sabil \\ ${ }^{1}$ UFR : APHE, Cadi Ayyad University, \\ Av. Prince My Abdellah, BP 2390 Marrakech, Morocco \\ Z. Benkhaldoun \\ ${ }^{1}$ UFR : APHE, Cadi Ayyad University, \\ Av. Prince My Abdellah, BP 2390 Marrakech, Morocco \\ M. Lazrek \\ ${ }^{1}$ UFR : APHE, Cadi Ayyad University, \\ Av. Prince My Abdellah, BP 2390 Marrakech, Morocco
}

\author{
O. Azagrouze \\ ${ }^{1}$ UFR : APHE, Cadi Ayyad University, \\ Av. Prince My Abdellah, BP 2390 Marrakech, Morocco
}

\begin{abstract}
As part of the Extremely Large Telescope (ELT) project, the sitetesting team at Morocco has measured from April 2008 to December 2009. The atmospheric properties at Aklim which was one of the candidates sites pre-selected.

In this paper we present the isoplanatic angle $\theta_{0}$ and the isopistonic angle $\theta_{\mathrm{p}}$ measurements at Aklim site. Statistics of the mentioned parameters are obtained from the whole data recorded using a Multi Aperture Scintillation Sensor (MASS) and a Differential Image Motion Monitor (DIMM) system. More representative results and statistics are shown hereafter.
\end{abstract}

\section{General Terms:}

Experimentation, interferometry, E-ELT

\section{Keywords:}

Instrumentation, atmospheric effects, MASS-DIMM , site testing

\section{INTRODUCTION}

As part of the European Extremely Large Telescope (E-ELT) project, several studies have been conducted to characterize a potential astronomical site for the next $42 \mathrm{~m}$ optical/near-infrared telescope (Vernin et al. 2011) [7]. The Aklim site in Morocco, is one of the four candidate sites for the E-ELT. It is located in the Moroccan Anti-Atlas mountains, at geographical coordinates $30^{\circ} 7^{\prime} 39^{\prime \prime}$ North and $08^{\circ} 18^{\prime} 31^{\prime \prime}$ West. It is a large plateau at an altitude of $2390 \mathrm{~m}$.

In a first step, our laboratory has conducted a preliminary prospecting campaign to measure the astronomical seeing, using a DIMM instrument (Sabil et al. 2010) [8]. The median and mean values of the seeing for the entire period of observation were 0.72 arcsec and 0.79 arcsec respectively. These preliminary results have indicated that Aklim has a favourable conditions for astronomical observations and could be a good site to host the next E-ELT.

In a second step, a MASS-DIMM system and a weather station were installed at Aklim site to measure other atmospheric parameters, such as, the isoplanatic angle $\theta_{0}$, the coherence time $\tau_{0}$ and the refractive index structure constant $C_{n}^{2}$. The results were published in (Vernin et al. 2011) [7]. In particular, the median isoplanatic angle measured at Akilm was 1.29 arcsec.

On the other hand, in stellar interferometry, there is an angle that is similar to the isoplanatic angle in adaptive optics, it's the isopistonic angle $\theta_{p}$. It is then an essential parameter for site characterization in interferometry when we want to study extra-galactic sources, that are generally weak sources.

Indeed, this angle plays an important role in off-axis cophasing of an interferometer, especially for detection and study the less luminous objects, is the goal of the instrument for Phase Referenced Imaging and Micro-arcsecond Astrometry (PRIMA) at the Very Large Telescope Interferometer (VLTI).

Indeed, for this type of observation luminous flux is so small that it is impossible to use it to make cophasing, The cophasing a refer- 
ence source other than scientific source has required as an alternative solution, This leads to two major constraints, the first is to find a source of bright star as reference to the cophasing and This is the problem of sky coverage (which will be studied in next paper). The second constraint, is more than the angular spacing between the reference and the target is large, more the correction error of the differential piston is important, named piston variance. Also and because of the lack of isopistonic measurements, many stud- ies of the piston effect have been carried out (Perrin 1997) [9]; (Quirrenbach et al. 1994) [11]. Recently, Esposito derived a compact expression of the differential piston variance (Esposito et al. 2000) [4]. This was done with the spatial correlation of Zernike modes of the wave front with a finite outer scale developed by Takato in (Takato et al. 1995) [10]. This expression requires the optical turbulence profile $C_{N}^{2}$ that are not available in literatures and that need specific instruments for their measurements. Moreover, calculations require a numerical integration that is not always a simple task.

Currently, there is no direct measurement of the isopistonic angle. In this paper we develop the analytical formula obtained in (Elhalkouj et al. 2007) [1] to infer $\theta_{p}$ from $\theta_{0}$, this work allows us to measure in the first one the isopistonic angle at Aklim site. This result will give us an idea of the magnitude of $\theta_{p}$, pending a direct measurement of this interferometric parameter in a next work.

Since the MASS system is not sensitive to the ground turbulence $(<500 \mathrm{~m})$, the global isoplanatic angle was calculated using both MASS and DIMM measurements and the Fried's parameter $r_{0}$ is directly deduced from DIMM measurements.

\section{THEORY}

\subsection{Isoplanatic angle}

The isoplanatic angle $\theta_{0}$ is the maximum angle between two directions which the atmospheric perturbations are correlated. Roddier et al. in [3] give the following expression:

$$
\theta_{0}=0.31 \frac{r_{0}}{H_{5 / 3}}
$$

with:

- $r_{0}$ is the FRIED parameter;

- $H_{5 / 3}$ is the averaging altitude related to the $C_{N}^{2}$ profile by the formula :

$$
H_{5 / 3}=\left[\frac{\int_{0}^{\infty} h^{5 / 3} C_{N}^{2}(h) \mathrm{d} h}{\int_{0}^{\infty} C_{N}^{2}(h) \mathrm{d} h}\right]^{3 / 5}
$$

\subsection{Isopistonic angle}

2.2.1 Effect of isopistonic angle on fringe visibility. For a two apertures interferometer, to study only the effect of differential piston we consider an ideal adaptive optic system. This lead as to assume that the only effect of the atmosphere is a constant offset with different values on the portions of wavefronts on each of the two apertures, which is called the differential piston.

The randomness of the differential piston fluctuations has an important effect on fringes contrast, and therefore the quality of interferometric measurements (Elhalkouj et al. 2007)[1].
2.2.2 Isopistonic versus isoplanatic angle. The effect of the differential piston must be corrected in real time. As in adaptive optics, this correction requires a reference star in most cases. Also, the angular separation between the target and the reference star must not be too large. The isopistonic angle $\theta_{p}$ is defined as the angular radius of a circular region where the anisopitonic error reduces the visibility in the image plane to no more than $80 \%$ of the unperturbed value (Esposito et al. 2000)[4] which corresponds to a residual piston error $\sigma_{p}$ about $\lambda / 10$.

In (Elhalkouj et al. 2007)[1] one gave a general definition for the isopistonic angle $\theta_{p}$ that corresponds to a residual piston error:

$$
\sigma_{p}\left(\theta=\theta_{p}\right)=\frac{\lambda}{n}
$$

where $\frac{\lambda}{n}$ is the desired accuracy for the fringe stabilization. The same study gave also the isopistonic angle $\theta_{p}$ as an analytical expression of atmospheric and instrumental parameters, for small and large apertures, as defined in (Elhalkouj et al. 2007)[1].

In this paper we will focus only on the case of small apertures. Indeed, it is in this context that the measurements were made in all the E-ELT candiadat sites.

$$
\theta_{p}(a)=\frac{2.3}{n} \theta_{0}\left(\frac{D}{r_{0}}\right)^{1 / 6} \frac{1}{\sqrt{P_{2}(a)}}, \quad a=\frac{\pi D}{L_{0}}
$$

$P_{2}(a)$ is a polynomial with the following expression

$$
\begin{gathered}
P_{2}(a)=0.216-0.225 a^{1 / 3}+0.122 a^{2}-0.096 a^{7 / 3} \\
+0.020 a^{4}-0.014 a^{13 / 3}+1.6410^{-3} a^{6} \\
-1.0210^{-3} a^{19 / 3}+7.9610^{-5} a^{8} \\
-4.6110^{-5} a^{25 / 3}
\end{gathered}
$$

\section{OBSERVATIONS AND DATA REDUCTION}

Data reported in this work were recorded at Aklim between April 2008 and December 2009. We have measured the isoplanatic angle over seventeen months. Many breakdowns have occurred during that period due to technical problems.

The combined Multi-Aperture Scintillation Sensor and Differential Image Motion Monitor (MASS/DIMM) device used during the EELT site testing campaign is described in detail in (Kornilov et al. 2007)[5]. The DIMM consists on the measurement of the variances of the longitudinal and transverse differential motions of two star's images on a small telescope focus (Sarazin \& Roddier 1990; Vernin \& Muñoz-Tuñón 1995) [6]. A set of 200 of $5 \mathrm{~ms}$ exposures time is used to obtain the two variances to deduce the integrated seeing $\varepsilon$ (Table 1). The MASS measures the isoplanatic angle $\theta_{0}$ and the free-seeing $\varepsilon_{f}$ of the free atmosphere $(h>500 \mathrm{~m})$ while the DIMM measures the whole integrated seeing. The MASS-DIMM system was installed on $5 \mathrm{~m}$ tower using a $0.35 \mathrm{~m}$ telescope.

is the exposure time, $\Delta t$ is the temporal resolution and $N_{\exp }$ is the number of exposures used to obtain a single variance measurement.

The free seeing $\epsilon_{\mathrm{f}}$ given by MASS is calculated by integrating the optical turbulence profile $C_{N}^{2}(h)$ from $0.5 \mathrm{~km}$ :

$$
\epsilon_{\mathrm{f}}=K_{1}\left(\int_{0.5}^{\infty} C_{N}^{2}(h) \mathrm{d} h\right)^{3 / 5}
$$


Table 1. Observation configuration of Aklim site. N-Data is the data number, $t_{\exp }$ is the exposure time, $\Delta t$ is the temporal resolution and $N_{\exp }$ is the number of exposures used to obtain a single variance measurement

\begin{tabular}{cccc}
\hline \multicolumn{4}{c}{ DIMM } \\
N-Data & $t_{\exp }(\mathrm{ms})$ & $\Delta t(\mathrm{~s})$ & $N_{\text {exp }} /$ samp \\
\hline 22289 & 5 & 42 & 200 \\
\hline \multicolumn{5}{c}{ MASS } \\
N-Data & $t_{\exp }(\mathrm{s})$ & $\Delta t(\mathrm{~s})$ & \\
\hline 17044 & 60 & 63 &
\end{tabular}

The hole seeing $\varepsilon$ mesured by the DIMM is given by:

$$
\epsilon=K_{1}\left(\int_{0}^{\infty} C_{N}^{2}(h) \mathrm{d} h\right)^{3 / 5}
$$

where $K_{1}=5.25 \lambda^{-1 / 5}$ and $\lambda=0.5 \mu \mathrm{m}$ is the wavelength and the measurements are then corrected by the zenith angle.

The isoplanatic angle $\theta_{\text {of }}$ (in the free atmosphere) measured by the MASS is calculated by integrating the turbulence profile with a $h^{5 / 3}$ weighting:

$$
\theta_{0 \mathrm{f}}=K_{2}\left(\int_{0.5}^{\infty} h^{5 / 3} C_{N}^{2}(h) \mathrm{d} h\right)^{-3 / 5}
$$

where $K_{2}=0.058 \lambda^{6 / 5}$.

The isoplanatic angle $\theta_{0}$ of the whole atmosphere is given by :

$$
\begin{aligned}
\theta_{0} & =K_{2}\left(\int_{0}^{\infty} h^{5 / 3} C_{N}^{2}(h) \mathrm{d} h\right)^{-3 / 5} \\
& =K_{2}\left(\int_{0}^{0.5} h^{5 / 3} C_{N}^{2}(h) \mathrm{d} h+\int_{0.5}^{\infty} h^{5 / 3} C_{N}^{2}(h) \mathrm{d} h\right)^{-3 / 5}
\end{aligned}
$$

Here we assume that the boundary turbulence is approximately represented by a single turbulent layer located at $h_{0}=250 \mathrm{~m}$ with a turbulent strength:

$$
\begin{aligned}
C_{N}^{2}\left(h_{0}\right) \delta h_{0} & =\int_{0}^{0.5} C_{N}^{2}(h) \mathrm{d} h \\
& =\left(\frac{\varepsilon}{K_{1}}\right)^{5 / 3}-\left(\frac{\varepsilon_{f}}{K_{1}}\right)^{5 / 3}
\end{aligned}
$$

thus:

$$
\theta_{0}=K_{2}\left[h_{0}^{5 / 3} C_{N}^{2}\left(h_{0}\right) \delta h_{0}+\left(\frac{\theta_{0 \mathrm{f}}}{K_{2}}\right)^{-5 / 3}\right]^{-3 / 5}
$$

All the follwing isopistonic values are calculated according to Eq 3 where $n=5$ (i.e, accuracy is equal to $\lambda / 5$ ), and the polynomial $P_{2}(a)$ is given by $\mathrm{Eq} 4$

Since the MASS-DIMM system does not measures the outer scale $L_{0}$, we have calculated the isopistonic angle for $L_{0}=25 \mathrm{~m}$ using equation 3

In Figure 1. we have presented an example of isopistonic angle evolution during the night of November 21, 2009. At the beginning of the night, the mean of this angle is about $22 \operatorname{arcsec}$ while

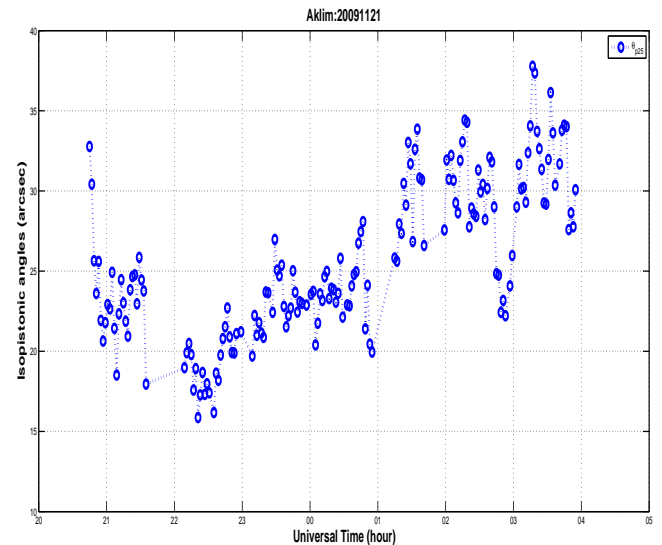

Fig. 1. Temporal evolution of the isopistonic angle at Aklim site during the night of November 21, 2009, for an outer scale of $25 \mathrm{~m}$.

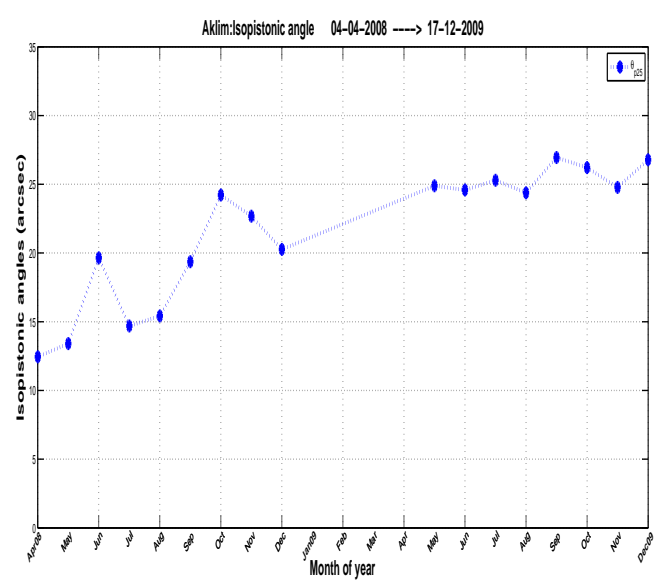

Fig. 2. Monthly evolution of the isopistonic angle at Aklim site.

it reached 30 arcsec at the end of the night. This is because the turbulence becomes weaker before sunrise.

In figure 2 we have presented the monthly averaged isopistonic angle for the entire observing period at Aklim site. The isopitonic angle median is $\theta_{\mathrm{P} 25}=25.31$ arcsec.

We can notice that during 2008 , the average of the isopistonic angle is smallar than the average over the next year. This may be explained by the fact that the wind was stronger in 2008 than in the 2009 by Sabil et al. 2010 [8].

We have shown in figure 3, the values averaged over the whole observation campaign versus Universal Time at Aklim. This figure shows that the isopistonic angle at Aklim is relatively stable during the night which is an important feature of an astronomical site. The figure 4 shows the histogram and cumulative frequeny of all isopistonic data at Aklim site. The median and mean values of the isopistonic angle for the entire period of observation are 26 arcsec and 25 arcsec respectively. 


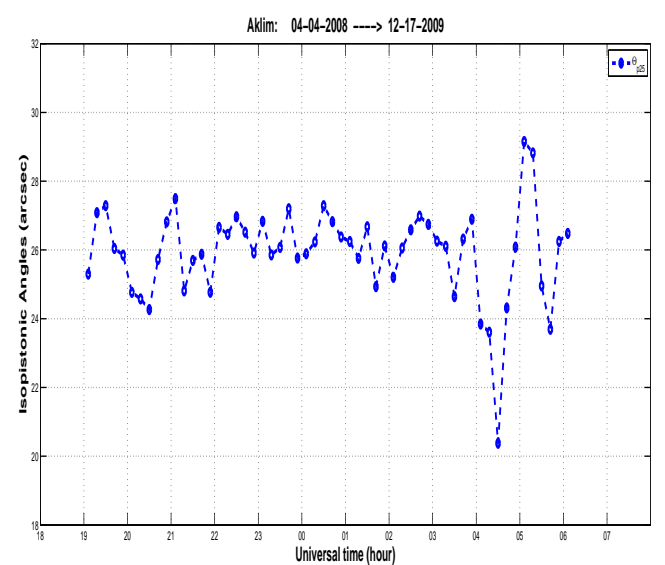

Fig. 3. Temporal evolution of the isopistonic angles at Aklim site during a average night.

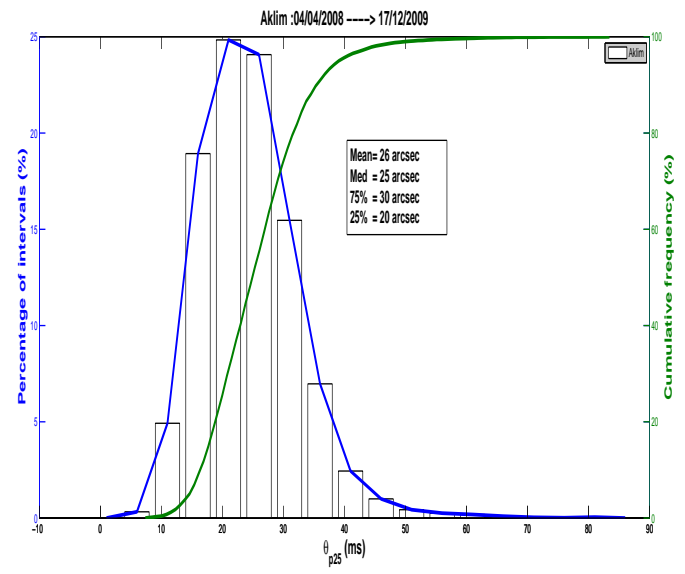

Fig. 4. Histogramme of all $\theta_{p}$ values for the entire observing period at Aklim site , the median and mean values are respectively 25 arcsec and 26 $\operatorname{arcsec}$.

\subsection{Comparison with other sites}

Let us compare Aklim site to Large Binocular Telescope (LBT) site for example. For an outer scale of $L_{0}=25 \mathrm{~m}$, an isoplanatic angle $\theta_{0}=1.4 \operatorname{arcsec}$ and a telescope diameter $\mathrm{D}=8.4 \mathrm{~m}$, the isopistonic angle at the LBT site is $\theta_{\mathrm{p}, \mathrm{LBT}}(K$ band $)=33.6$ arcsec (Elhalkouj et al. 2007)[1]. At Aklim site, the mean of the isoplanatic angle is $: \theta_{0, \mathrm{Aklim}}(V$ band $) \simeq 1.43$ arcsec. So $\theta_{0, \mathrm{Aklim}}(K$ band $) \simeq 7.7$ arcsec. Using equation (3), we obtain the isopistonic angle value $\theta_{\mathrm{p}, \mathrm{Aklim}}(K$ band $) \simeq 35.23$ arcsec. This means that Aklim site has the comparable qualities as other well known interferometric sites.

\section{CONCLUSION}

As it was pointed out at the beginning of this paper, the isopistonic angle, in interferometry, plays a similar role to that played by the isoplanatic angle in adaptive optics.

Moreover, this angle plays an important role for other project in progress as PRIMA (Phase-Referenced Imaging and Micro- arcsecond Astrometry) facility of the Very Large Telescope Interferometer. A major goal of this project is to perform differential narrow-angle astrometry with accuracy of $10 \mu$ as (Deplancke et al. 2007)[2]. Indeed we must take into account the isopistonic domain to measure these small angles. The consideration from above shows the importance that has the isopistonic angle in Deep sky and ExtraGalactic studies.

This work is a complement of the study of Aklim site within the E-ELT project. As we know, the site has not been chosen finally to host the E-ELT, but it is a potential site for astronomical observations.

An experiment dedicated to the measurement of the isopistonic angle is one of our major themes.

\section{ACKNOWLEDGMENTS}

This work is supported by the collaboration with European Union by mean of the FP6 program. We are also grateful to the Moroccan Hassan II Academy of Science and Technical which supported financially the site testing campaigns at Aklim site.

\section{REFERENCES}

[1] Elhalkouj T., Ziad A., Petrov R. , Lazrek M., Elazhari Y and Benkhaldoun Z., 2007, Isopistonic angle for multi aperture interferometers from isoplanatic angle, A\&A, 477, 337-344

[2] Delplancke F., 2007, The PRIMA facility Phase-Referenced Imaging and Micro-arcsecond Astrometry, ESO, Karl Schwarschildstrasse 2, Garching bei Munchen, Germany (5 December 2007)

[3] Roddier F., Gilli J.M, Vernin J., On the isoplanatic patch size in stellar speckle interferometry, 1982, J.Optic.13, 63

[4] Esposito S., Riccardi A., Femenía B., Differential piston angular anisoplanatism for astronomical optical , 2000, Astron. Astrophys. 353, L29L32

[5] Kornilov V., Tokovinin A., Shatsky N., Voziakova O., Potanin S. and Safonov, B., Combined MASS-DIMM instruments for atmospheric turbulence studies, 2007, MNRAS. 382, 1268

[6] Sarazin M., and Roddier F., The ESO differential image motion monitor, Astronomy \& Astrophysics, 1990 , 227, 294 .

[7] Vernin, J., Muñoz-Tuñón, C., Sarazin, M., Benhida, S., Benkhaldoun, Z., Delgado, J.M., Fuensalida, J.J., Hach, Y., Lazrek, M., Lombardi, G., Navarrete, J., Recabarren, P., Reyes, M., Sabil, M., Trinquet, H.,Varela, A.V and Ramio, V., Astronomy \& Astrophysics manuscript no 20111503 overiew , 2011.

[8] Sabil, M., Benkhaldoun, Z., Lazrek, M., Benhida, A., Hach, Y., Habib, A. and Bounhir, A., First characterization of Jbel Aklim in Moroccan Anti-Atlas as a potential site for the E-ELT. Meteorological parameters and seeing measurements, A\&A, 69, 522, 2010

[9] Perrin, G. 1997, A\&AS, 121, 553 Quirrenbach, A., Mozourkurkewich, D., Busher, D. F., et al. 1994, A\&A, 286, 1019

[10] Takato, N., \& Yamaguchi, I. 1995, J. Opt. Soc. Am. Opt. Image Sci. Vision, 12, 958

[11] Quirrenbach, A., Mozourkurkewich, D., Busher, D. F., et al. 1994, A\&A, 286, 1019 